\title{
Bim, Bad, and Bax: a deadly combination in epileptic seizures
}

\author{
Jerome Niquet ${ }^{1,2}$ and Claude G. Wasterlain ${ }^{1,2,3}$
}

\begin{abstract}
'Epilepsy Research Laboratory, Veteran's Administration Greater Los Angeles Healthcare System, West Los Angeles, California, USA. ${ }^{2}$ Department of Neurology and ${ }^{3}$ Brain Research Institute, David Geffen School of Medicine, University of California at Los Angeles, Los Angeles, California, USA.
\end{abstract}

\begin{abstract}
Several Bcl-2 family members, including Bim, may contribute to programmed cell death by inducing mitochondrial cytochrome $c$ release, which activates caspase-9 and then caspase-3, the "executioner" of the cell. In this issue of the JCI, Shinoda and collaborators show the key role of Bim in epileptic seizure-induced neuronal injury and identify the contribution of transcription factors responsible for seizure-induced Bim upregulation (see the related article beginning on page 1059).
\end{abstract}

Neuronal death following brain insults often results from the execution of cellular programs that resemble those involved in developmentally programmed cell death. Cell demise is often brought about by the activation of caspases, a family of cysteine proteases. When what is usually called the "extrinsic" pathway of programmed cell death is induced, the first step involves the activation of extracellular cell death receptors of the TNF superfamily, which recruit other proteins to form a complex that activates caspase-8, which in turn activates caspase-3 (Figure 1) (1). This “executioner" caspase kills the cell through its widespread proteolytic effects, activating DNA breakdown, inactivating DNA repair enzymes, and attacking the cytoskeleton, among other activities. In the "intrinsic" pathway of programmed cell death, the mitochondrion plays a critical role by releasing cytochrome $c$ from its intermembrane space to the cytosol, where, in association with apoptotic protease-activating factor- 1 and dATP, it forms the apoptosome complex, activating caspase-9, which in turn activates caspase-3 (Figure 1) (1). Cytochrome $c$ release is regulated by the Bcl-2 family of proteins, which are pro- or antiapoptotic, depending on their ability to promote or suppress its

Nonstandard abbreviations used: apoptopsis-inducing factor (AIF); Bcl-2-associated death promoter (Bad); $\mathrm{Bcl}-2$-associated $\mathrm{X}$ protein (Bax); Bcl-2-interacting mediator of cell death (Bim); $\mathrm{BH} 3$ interacting domain death agonist (Bid); forkhead in rhabdomyosarcoma (FKHR); FKHR-like-1 (FKHRL-1); kainic acid (KA); status epilepticus (SE).

Conflict of interest: The authors have declared that no conflict of interest exists.

Citation for this article:

J. Clin. Invest. 113:960-962 (2004)

doi:10.1172/JCI200421478. release. The balance between proapoptotic factors (BH3-interacting domain death agonist [Bid], Bcl-2-associated death protein [Bad], Bcl-2-interacting mediator of cell death [Bim], Bcl-2-associated $\mathrm{X}$ protein [Bax], and $\mathrm{Bcl}-2$ homologous antagonist killer [Bak]) and antiapoptotic factors (Bcl-2, Bcl-x, and Bcl-w) determines cytochrome $c$ release and the fate of the cell (1). This balance depends not only on the respective levels of expression of these factors but also on their post-translational modifications and interactions (1).

\section{Seizures induce the "intrinsic" pathway of the cell death program}

Henshall and collaborators use a model of status epilepticus (SE; severe repetitive epileptic seizures) induced by injection of kainic acid (KA) into the rat amygdala. In rats with $\mathrm{KA}$-induced $\mathrm{SE}$, the authors observed all the key elements of "intrinsic" pathway induction: cytochrome $c$ release; apoptosome formation; caspase- 9 and caspase-3 activation (Figure 1); neuroprotection by caspase- 9 and caspase- 3 inhibitors; and double-stranded DNA breaks $(2,3)$. In rats with KA-induced SE, Bad was dissociated from chaperone protein 14-3-3, which allowed it to dimerize with Bcl-xl. Bax, displaced from Bcl-xl binding, translocated to the mitochondria, causing release of cytochrome $c$ and activation of caspase-9 and caspase-3. Administration of the calcineurin inhibitor FK506 was neuroprotective, possibly by blocking Bad dephosphorylation and preventing its dissociation from 14-3-3 (4). However, in an in vitro model of SE, FK506 did not prevent cell death or caspase-3 activation (5), suggesting that Bad's role was secondary. In this issue of the JCI, the same group attempts to identify the key proteins controlling cytochrome $c$ and apoptotic death in the same KAinduced model of SE and reports that Bim, which is usually sequestered in the endoplasmic reticulum-dynein complex, was released from that complex and from association with 14-3-3 (6). Immunoprecipitation experiments suggest that Bim and Bcl-w form an oligomer (6), presumably releasing $\operatorname{Bax}(7)$, which then translocates to the mitochondria (4). Shinoda et al. now report that hippocampal Bim expression was upregulated by seizures. In the in vitro seizure model, neuronal survival increased when Bim expression was suppressed by Bim antisense oligonucleotides, suggesting that the Bim pathway had a key role in seizure-induced cell death (6). However, in a different seizure model (in which SE is induced in rats by intraventricular KA administration, which might have direct toxic effects), Korhonen et al. showed that the Bim pathway did not contribute to hippocampal injury, as Bim expression was rapidly downregulated (8).

\section{Upstream control mechanisms: role of the transcription factors}

Shinoda et al. (6) investigated the upstream mechanisms of Bim upregulation and cytochrome $c$ release. Bim expression may be controlled by transcription factors of the forkhead in rhabdomyosarcoma (FKHR) family, including FKHR and FKHR-like-1 (FKHRL-1) (9). In the KAinduced model of SE, the authors showed a downregulation of the phosphorylated (inactive) forms of FKHR and FKHRL-1, suggesting that their unphosphorylated (active) forms were upregulated and translocated to the nucleus, where they upregulated Bim expression. In vitro, epileptic seizure-like activity increased binding of FKHR to the Bim promoter. Blocking the dephosphorylation of FKHR and/or FKHRL-1 with sodium orthovanadate improved the survival of hippocampal neurons. In the cortex of the same ani- 


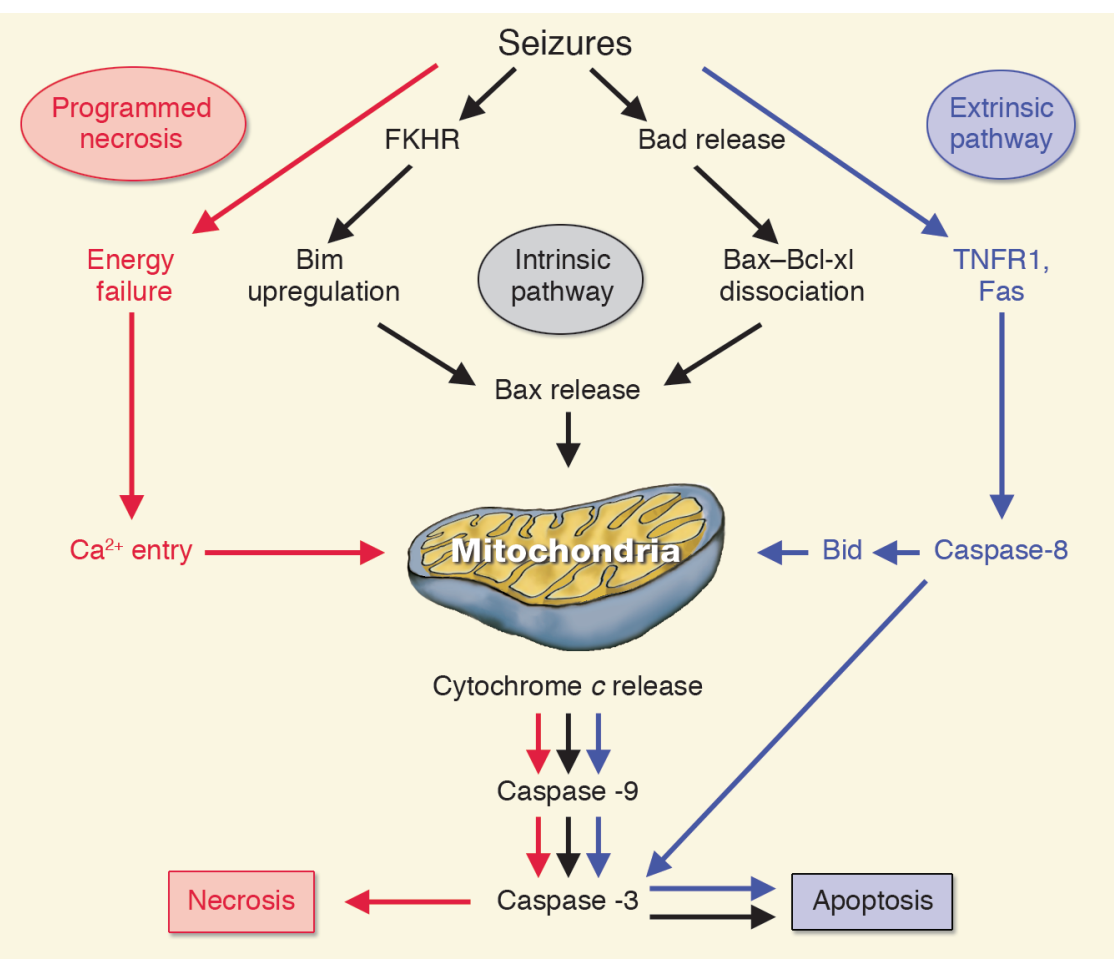

Figure 1

Putative neuronal death pathways induced by epileptic seizures. In the "extrinsic" pathway of programmed cell death, activation of extracellular cell death receptors of the TNF superfamily (Fas and tumor necrosis factor receptor 1 [TNFR1]) induces activation of catalytic enzymes caspase-8 and caspase-3, causing widespread proteolytic damage and cell death. Mitochondria have a central role in the other forms of programmed cell death. In the "intrinsic" pathway, cytochrome $c$ release is triggered by translocation of the proapoptotic factor Bax to the mitochondria. Seizures induce dephosphorylation of transcription factors FKHR and FKHRL-1, leading to the upregulation of the proapoptotic factor Bim, which forms an oligomer with Bcl-w, triggering Bax activation. Seizures may also induce dissociation of Bad from chaperone protein 14-3-3. Released Bad binds to Bcl-xl, provoking dissociation of Bax-Bcl-xl and Bax release. Bax penetrates the mitochondrial outer membrane and triggers cytochrome $c$ release from the intermembrane space to the cytosol, where it activates caspase-9, which then activates caspase- 3 . Alternatively, cytochrome $c$ release may be induced after Bid truncation by active caspase-8, linking the "extrinsic" and "intrinsic" pathways. In "programmed necrosis," seizures deplete energy reserves, leading to mitochondrial calcium overloading, opening of the mitochondrial transition pore, and/or mitochondrial swelling and rupture of the outer membrane. Subsequent cytochrome $c$ release activates the caspase cascade and leads to cell demise.

mals, the PI3K inhibitor LY294002, which prevents Akt activation, induced FKHR translocation, upregulated Bim expression, and increased cell death, suggesting a protective role for the Akt pathway, as seen in ischemic tolerance (10). In human temporal lobes ablated for intractable epilepsy, the authors observed Akt activation, upregulation of the inactive form of FKHR, and Bim downregulation (compared with autopsy samples). These results were interpreted as a kind of "epileptic tolerance": Akt activation may have phosphorylated and inactivated FKHR, reducing Bim expression and cell death, suggesting that Akt activation may be con- sidered as a potential therapeutic avenue. However, the possibility that decreased Bim immunoreactivity may reflect neuronal loss has not been ruled out.

\section{A work in progress}

The authors' elegant approach enhances our understanding of neuronal death pathways following epileptic seizures (6). However, the beautiful tapestry they have weaved is far from complete; there are still holes to fill. For example, the upregulation and nuclear translocation of active FKHR in the injured hippocampus has not been explicitly demonstrated (6). The neuroprotective effect of sodium orthovanadate (6), which is not a specific inhibitor, might be due to inhibition of a phosphatase that is not part of the Bim pathway. The assumption that cell death mechanisms involved in the in vitro and in vivo seizure models are similar might be optimistic: FK506 is neuroprotective in the in vivo model (4) and not in the in vitro model (5).

\section{Cross-talk between pathways}

In summary, the studies from Shinoda et al. (6) suggest a central role for mitochondria in the process of epileptic seizure-induced cell death. Specific death effector proteins (Bim in KA-induced SE, but also Bid and/or $\mathrm{Bad}$; Bad in ischemia, but perhaps with Bid and/or Bim) may initiate this pathway (1, 11). Once the cell suicide program has been initiated, however, pathway specificity is replaced by redundancy, and "promiscuous" caspases activate each other through multiple feedback loops. For example, the initiator caspases 8 and 9 activate the executioner caspases 3 and 7. Active caspase-3, in turn, cleaves caspases 2, 6, 8, 10, and perhaps 9, providing a tremendous amplification loop (1). Caspase- 8 cleaves Bid, which releases cytochrome $c$, bridging the "extrinsic" and "intrinsic" pathways (11). This might explain why specific caspase inhibitors rarely achieve complete protection from neuronal injury and might prompt us to question the wisdom of the quest for inhibitor specificity.

Another conclusion from those studies $(1-6,11)$ is that many key elements of the "extrinsic" and "intrinsic" pathways of programmed cell death are constitutively expressed, and therefore their de novo synthesis is not required in order for these proteins to play an active role in neuronal death. Post-translational modifications (e.g., phosphorylation and dephosphorylation) and protein-protein interactions (e.g., binding, dimerization, and oligomerization) play a crucial role in the execution of the cell death program (1). This system is less energy dependent than traditional apoptotic cascades and appears more compatible with conditions such as SE, which usually entail some degree of energy failure.

\section{Putative pathways of SE-induced neuronal death}

Where do these pathways stand in the overall spectrum of mechanisms underlying neuronal death? We can identify at least four separate pathways involving neuronal death programs. The "extrinsic" pathway, which does not require mitochondrial par- 
ticipation, and the "intrinsic" pathway, which does, offer many alternative routes of caspase activation culminating in a relatively slow, caspase-dependent death with apoptotic morphology (1). Rapid death with a necrotic morphology can result from direct release of cytochrome $c$ from calcium-overloaded, energy-depleted mitochondria, followed by activation of caspase- 9 and -3. Such "programmed necrosis" is seen after hypoxic/ischemic neuronal necrosis in primary cultures of dentate gyrus (12). It may also contribute to neuronal death from SE in the adult brain (13), which is characterized by a predominantly necrotic morphology (14) and by calcium overload of neuronal mitochondria (15; Figure 1). Another program involves apoptosis-inducing factor (AIF), which, when released from mitochondria, triggers a cell death pathway that does not require caspase activation (16) and may produce a necrotic appearance (17). This "short list" of death pathways will undoubtedly grow, as it ignores the less well-defined roles of Smac/Diablo, endonuclease $\mathrm{G}$, calpains, cathepsins, and many other important factors (1). It is a measure of our semantic confusion that TNF is the classical trigger of the "extrinsic" pathway of apoptosis and that AIF-mediated death may often look necrotic.
Address correspondence to: Jerome Niquet, Epilepsy Research Laboratory, Research 151, Veterans Affairs Greater Los Angeles Healthcare System, 11301 Wilshire Boulevard, West Los Angeles, California 90073, USA. Phone: (310) 478-3711 ext. 41974; Fax: (310) 268-4856; E-mail: jniquet@ucla.edu.

1. Liou, A.K.F., Clark, R.S., Henshall, D.C., Yin, X.M., and Chen, J. 2003. To die or not to die for neurons in ischemia, traumatic brain injury and epilepsy: a review on the stress-activated signaling pathways and apoptotic pathways. Prog. Neurobiol. 69:103-142.

2. Henshall, D.C., Chen, J., and Simon, R.P. 2000. Involvement of caspase-3-like protease in the mechanism of cell death following focally evoked limbic seizures. J. Neurochem. 74:1215-1223.

3. Henshall, D.C., et al. 2001. Formation of the Apaf-1/ cytochrome $c$ complex precedes activation of caspase-9 during seizure-induced neuronal death. Cell Death Differ. 8:1169-1181.

4. Henshall, D.C., et al. 2002. Activation of Bcl-2-associated death protein and counter-response of Akt within cell populations during seizure-induced neuronal death. J. Neurosci. 22:8458-8465.

5. Meller, R., et al. 2003. Seizure-like activity leads to the release of BAD from 14-3-3 protein and cell death in hippocampal neurons in vitro. Cell Death Differ. 10:539-547.

6. Shinoda, S., et al. 2004. Bim regulation may determine hippocampal vulnerability after injurious seizures and in temporal lobe epilepsy. J. Clin. Invest. 113:1059-1068. doi:10.1172/JCI200419971.

7. Wilson-Annan, J., et al. 2003. Proapoptotic BH3-only proteins trigger membrane integration of prosurvival Bcl-w and neutralize its activity. J. Cell Biol.162:877-888.

8. Korhonen, L., Belluardo, N., Mudo, G., and Lind- holm, D. 2003. Increase in Bcl-2 phosphorylation and reduced levels of $\mathrm{BH} 3$-only $\mathrm{Bcl}-2$ family proteins in kainic acid-mediated neuronal death in the rat brain. Eur. J. Neurosci. 18:1121-1134.

9. Burgering, B.M., and Kops, G.J. 2002. Cell cycle and death control: long live Forkheads. Trends Biochem. Sci. 27:352-360.

10. Yano, S., et al. 2001. Activation of Akt/protein kinase $\mathrm{B}$ contributes to induction of ischemic tolerance in the CA1 subfield of gerbil hippocampus. J. Cereb. Blood Flow Metab. 21:351-360.

11. Henshall, D.C., et al. 2001. Cleavage of bid may amplify caspase-8-induced neuronal death following focally evoked limbic seizures. Neurobiol. Dis. 8:568-580.

12. Niquet, J., Baldwin, R.A., Allen, S.G., Fujikawa, D.G., and Wasterlain, C.G. 2003. Hypoxic neuronal necrosis: protein synthesis-independent activation of a cell death program. Proc. Natl. Acad. Sci. U. S. A. 100:2825-2830.

13. Niquet, J., Liu, H., and Wasterlain, C.G. 2004. Programmed neuronal necrosis and status epilepticus. Epilepsia. In press.

14. Fujikawa, D.G., Shinmei, S.S., and Cai, B. 2000. Kainic acid-induced seizures produce necrotic, not apoptotic, neurons with internucleosomal DNA cleavage: implications for programmed cell death mechanisms. Neuroscience. 98:41-53.

15. Griffiths, T., Evans, M.C., and Meldrum, B.S. 1983. Intracellular calcium accumulation in rat hippocampus during seizures induced by bicuculline or L-allylglycine. Neuroscience. 10:385-395.

16. Cande, C., Cecconi, F., Dessen, P., and Kroemer, G. 2002. Apoptosis-inducing factor (AIF): key to the conserved caspase-independent pathways of cell death? J. Cell Sci. 115:4727-4734.

17. Yu, S.W., Wang, H., Dawson, T.M., and Dawson, V.L. 2003. Poly(ADP-ribose) polymerase- 1 and apoptosis inducing factor in neurotoxicity. Neurobiol. Dis. 14:303-317. 